\title{
HOXB13 expression in metastatic prostate cancer
}

\author{
Justine Varinot $^{1} \cdot$ Eva Comperat $^{1}$
}

Received: 21 April 2016/Accepted: 26 April 2016/Published online: 16 May 2016

(C) Springer-Verlag Berlin Heidelberg 2016

Sir,

We would like to thank Barresi et al. for the interest shown in our publication in Virchows Archiv [1]. In a recent study, our group analyzed immunohistochemical expression of HOXB13 in a series of metastatic tumors from different known primaries, including 15 metastases from prostate cancer [1]. We are pleased that the results Barresi et al. reported in their recent paper are close to ours, notably strong staining for HOXB13 in metastatic tumor tissue [2]. They encountered the same technical problems as we did, and we fully agree with their findings. With Barresi et al., we find that more specific additional markers should be used to confirm bone metastasis originating from a prostate cancer. This topic has only rarely been addressed in formal studies, mostly due to the problem of small samples which are difficult to cut and stain. In conclusion, we emphasize that determining prostate cancer origin of a bone metastasis has been insufficiently explored in terms of methodology, and additional studies would be very welcome.

\section{References}

1. Varinot J, Furudoï A, Drouin S, Phe V, Penna RR, Roupret M, Bitker MO, Cussenot O, Compérat E. HOXB13 protein expression in metastatic lesions is a promising marker for prostate origin. Virchows Arch. 2016.

2. Barresi V, Ieni A, Cardia R, Licata L, Vitarelli E, Reggiani Bonetti L, G. T (2016 Mar) HOXB13 as an immunohistochemical marker of prostatic origin in metastatic tumors. APMIS 124(3):188-193
Justine Varinot

justinevarinot@hotmail.com

1 Department of Pathology, Hopital Pitie-Salpetriere, 47-83, Boulevard de l'Hopital, 75013 Paris, France 